\title{
Programa de intercambio entre operadores de telecentros: una nueva generación de redes en el movimiento de telecentros panamericano.
}

\author{
Oscar Maeso \\ Fundación ChasquiNet en Quito (Ecuador) \\ $<$ oscar@chasquinet.org >
}

\begin{abstract}
Resumen
¿Puede una red ir más allá del intercambio de información? ¿Cómo puede telecentro de Chile beneficiarse del trabajo que realiza otro telecentro en México?

El Programa de Intercambio de Telecentros es un proyecto diseñado e implementado por la Alianza de Telecentros de las Americas (TAP) que ha demostrado que las redes de conocimiento pueden y deben evolucionar hacia redes en las que sus miembros sean capaces de elaborar $e$ implementar proyectos de forma conjunta y colaborativa. A lo largo de este artículo se analiza este interesantísimo proyecto que fue ejecutado en el año 2006.
\end{abstract}

\section{Redes para la elaboración de proyectos conjuntos.}

Un boleto de avión comprado en Ecuador para un viaje desde México Distrito Federal a Santiago de Chile. El billete se gestiona a través de Internet por los coordinadores del PIT que trabajan en la Fundación ChasquiNet en Quito; el boleto se emite a nombre de Juan Carlos Rico Campos, el Subdirector de Centros Interactivos Poder Joven de México, que presentó su propuesta de intercambio conjuntamente con la Subtel (Subsecretaria de telecomunicaciones) de Chile..

Los proyectos basados en red nos muestran un mundo sin fronteras donde los resultados y los actores son globales, donde se comparte algo más que información digital para alcanzar objetivos comunes.

Las redes electrónicas o digitales han mostrado su eficacia como redes de apoyo, donde los distintos actores comparten e intercambian información para fortalecer determinados procesos. Un ejemplo de este tipo de redes es somos@telecentros, una red de telecentros con más de 3.500 miembros activos que hasta la fecha ha sido el lugar de encuentro para responsables de telecentros, operadores, investigadores, estudiantes y otros actores del movimiento de telecentros que han utilizado las distintas herramientas electrónicas disponibles (listas de distribución, centros de recursos, blogs, etc.) para apoyarse mutuamente.

El Consorcio de Telecentros de las Americas $\left(\mathrm{TAP}^{1}\right)$ a través del PIT ha dado una vuelta de tuerca al concepto de redes y ha demostrado la posibilidad de que estas sean un espacio adecuado para trabajar a distancia en la consecución de objetivos comunes. Una evolución de una red informativa a una red transformadora y activa que se analiza mediante el estudio de caso del PIT.

\section{Sobre los actores}

TAP, es una red de redes que representa aproximadamente a 10.000 telecentros y representantes del movimiento de telecentros a lo largo del continente americano (Norte América, Centro América, Sudamérica y el Caribe).

Los miembros fundadores de TAP se muestran en la siguiente tabla.

\begin{tabular}{|l|l|l|l|}
\hline Nombre & Año de inicio & Breve descripción & Web \\
\hline Somos@Telecentros & 1999 & $\begin{array}{l}\text { Red de telecentros y redes de } \\
\text { telecentros que representa 3.750 } \\
\text { telecentros a lo largo de América Latina }\end{array}$ & \\
\hline
\end{tabular}




\begin{tabular}{|c|c|c|c|}
\hline & & y el Caribe & \\
\hline Aspira Association, Inc & 1961 & $\begin{array}{l}\text { Red de } 135 \text { telecenters al servicio de las } \\
\text { comunidades latinas en Estados Unidos } \\
\text { y Puerto Rico }\end{array}$ & www.aspira.org \\
\hline $\begin{array}{l}\text { Community Technology } \\
\text { Centers' Network (CTCNet) }\end{array}$ & 1989 & $\begin{array}{l}\text { Red de mas de } 1.000 \text { telecentros } \\
\text { independientes (centros comunitarios } \\
\text { tecnológicos) que facilita acceso a } \\
\text { computadoras, el Internet y otras } \\
\text { herramientas tecnológicas a los } \\
\text { residentes de comunidades de bajos } \\
\text { ingresos a lo largo de los Estados } \\
\text { Unidos }\end{array}$ & www.ctcnet.org \\
\hline $\begin{array}{lr}\text { Pacific } & \text { Community } \\
\text { Networks } & \text { Association } \\
\text { (PCNA) } & \end{array}$ & 1993 & $\begin{array}{l}\text { Organización de } 28 \text { redes locales que } \\
\text { agrupan a } 560 \text { telecentros en la } \\
\text { Columbia Británica y el Yukon } \\
\text { (Canadá). Adicionalmente, PCNA } \\
\text { representa actualmente a } 4,500 \\
\text { telecentros y otras redes comunitarias } \\
\text { en el consorcio de TAP }\end{array}$ & www.pcna.ca \\
\hline $\begin{array}{l}\text { Association for Community } \\
\text { Networking }\end{array}$ & & $\begin{array}{l}\text { Association for Community } \\
\text { Networking facilita recursos, } \\
\text { aprendizaje compartido y asistencia con } \\
\text { mucha experiencia para ayudar a las } \\
\text { comunidades y organizaciones a usar } \\
\text { las tecnologías de comunicación e } \\
\text { información de forma efectiva }\end{array}$ & \\
\hline Fundación ChasquiNet & 1997 & $\begin{array}{l}\text { Fundación que promueve el uso } \\
\text { estratégico de las TIC en los sectores } \\
\text { sociales de los países en vías de } \\
\text { desarrollo a través de la acción práctica, } \\
\text { la investigación, las políticas, la } \\
\text { educación y la mejora de la } \\
\text { comunicación. El objetivo de la } \\
\text { Fundación ChasquiNet es contribuir al } \\
\text { uso de las TIC como un mecanismo de } \\
\text { apoyo para el desarrollo y mejora de las } \\
\text { condiciones de vida, la educación y el } \\
\text { fortalecimiento de la cultura de las } \\
\text { personas. }\end{array}$ & www.chasquinet.org \\
\hline
\end{tabular}

Tabla 1: Miembros fundadores de TAP.

Los telecentros son concebidos por TAP como espacios comunitarios de transformación social y desarrollo humano dónde los habitantes de las comunidades menos favorecidas tienen acceso a las Tecnologías de Información y Comunicación (TIC) y las utilizan como herramientas de apoyo a sus procesos de desarrollo humano y social.

Bajo este concepto se creó TAP, con el objetivo de incrementar la variedad y la calidad de apoyo existente en torno a los telecentros para que estos a su vez puedan servir más efectivamente a sus respectivas comunidades.

Para alcanzar estos objetivos TAP considera que uno de de los primeros factores que ayuda a crecer y que apoya la eficacia y la sostenibilidad de los telecentros es la capacidad para compartir conocimiento, ideas y modelos de programa. A lo largo de este artículo utilizaremos el término redes de conocimiento para describir el medio por el que entendemos que se facilitan dichos procesos tanto en el caso del PIT de TAP como en cualquier otra red humana que utiliza medios, sean estos, físicos y/o virtuales como medios de transmisión e intercambio de información y para su transformación en conocimiento. 
Los operadores de los telecentros son las personas encargadas de guiar, capacitar y fortalecer a las comunidades en el uso estratégico de las TIC para mejorar sus procesos de desarrollo. En muchos casos el/la operador/a es el/la encargado/a de definir los contenidos de los talleres e identificar las necesidades de capacitación de la comunidad. Por lo tanto la labor de los/as operadores/as es clave y fundamental. En este sentido son muchos los autores que señalan la importancia de estos actores para lograr la efectividad y la sostenibilidad de los telecentros (DELGADILLO 2002, PROENZA 2004, ROESSNER 2005).

El PIT que analizamos en este artículo, es un proyecto conceptualizado e implementado por TAP que cuenta con el apoyo financiero de telecentre.org ${ }^{2}$. El proyecto fue formulado ante una necesidad detectada en las bases del movimiento de telecentros y que se refiere a la casi total inexistencia de encuentros físicos entre los operadores de base de los telecentros. Si bien existen numerosos encuentros internacionales sobre el uso de las TIC para el desarrollo (TICpD), los operadores y las bases del movimiento telecentrista no han podido participar en estos fundamentalmente por las razones que se detallan a continuación:

- El coste de los viajes.

- Las barreras del lenguaje.

- Escaso valor de la mayor parte de las conferencias internacionales para las bases del movimiento telecentrista. (Falta de adaptación a sus necesidades).

La consecuencia es que son muy pocos los operadores de base de telecentros de las diferentes regiones los que han tenido la oportunidad de estar juntos físicamente e intercambiar sus experiencias cara a cara.

Una red de conocimiento, como hemos visto, puede utilizar sistemas electrónicos para el intercambio de información, por ejemplo el correo electrónico; pero sino existen encuentros físicos que permitan a los distintos actores conocerse, es muy difícil que surjan las dimensiones humanas tales como la confianza, el entendimiento mutuo y compartir una visión común, elementos que TAP considera necesarios para la construcción colectiva de conocimiento a través de las redes humanas.

Para que los operadores puedan desarrollar su verdadero potencial deben tener una capacitación multidisciplinar y continua. Es aquí donde las redes de conocimiento, y en especial los medios virtuales, juegan un papel muy importante, facilitando un medio de intercambio de conocimiento y capacitación eficiente de bajo coste y adaptado a las necesidades de estos actores.

A los actores de PIT debemos incluir uno menos evidente pero de creciente importancia: las comunidades transnacionales (CANALES, ZLONISKI 2000) que representan una activación a través de la migración de diversos factores y procesos de articulación en el ámbito cultural, social y económico, entre comunidades e instituciones sociales distantes y separadas geográficamente. Estas comunidades dispersas geográficamente, utilizan las TIC para comunicarse y mantener sus vínculos. Los telecentros están siendo utilizados por las comunidades latinas no solo como puntos de acceso a las TIC sino como lugar de encuentro con las comunidades de inmigrantes en sus países de destino. Por lo tanto los actores van mas allá de los participantes directos (operadores/as de telecentros), existen comunidades locales vinculadas con otros lugares distantes a través de las comunidades transnacionales, algunas comunidades antes aisladas son cada vez más globales y han hecho aparecer nuevos términos como la glocalidad que representa nuevos fenómenos surgidos de la conjunción de lo local y lo global. El PIT tal y como se detalla en este articulo, demuestra el potencial de las redes como instrumentos para beneficiar a un gran numero de personas mediante la elaboración de proyectos colaborativos basados en red.

\section{Una evolución en el movimiento de telecentros}

La convergencia entre las telecomunicaciones y la informática es un elemento fundamental para la aparición de la denominada sociedad de la información, una sociedad marcada por un gran dinamismo. Entre otros elementos este dinamismo ha producido una serie de cambios en los últimos años entre los que destacamos la evolución de una Internet estática a una Internet dinámica; la transformación de una red de maquinas a una red de personas o red social conocida como Web 2.0; las tecnologías que utilizamos se encuentran también en una evolución constante, las aparición de las redes inalámbricas ha facilitado y abaratado la conectividad permitiendo entre otras cosas que los telecentros florezcan en lugares cada vez más remotos del continente americano. Todos estos cambios se han dado en apenas una década lo cual nos da muestra de la rapidez con la que las TIC evolucionan. El movimiento de telecentros no ha sido ajeno a estos cambios que poco a poco han sido incorporados de una $\mathrm{u}$ otra manera.

La evolución de las redes a las que ha contribuido el PIT, es el elemento que analizamos en este artículo. Este ha sido un proyecto piloto fruto de la experiencia de todos los miembros de TAP en el trabajo con telecentros a lo largo del continente americano. Las redes que conforman el movimiento de telecentros de las americas, hasta ahora no habían explorado la posibilidad de realizar proyectos colaborativos basados en red, sin embargo el PIT ha abierto esta posibilidad en el movimiento y como veremos lo ha hecho con muy buenos resultados. 
Una red electrónica (GUIJT y WOODHILL, 2005) es un grupo de personas que interactúan a través de los medios de Internet y están vinculadas por un interés común (en nuestro caso el movimiento de telecentros. Internet, entre otras cosas, ofrece lo siguiente:

- Vías económicas y rápidas para la comunicación. (en la actualidad fundamentalmente escrita)

- Intercambio rápido y facilitado o moderado de diálogo escrito sobre asuntos o temas relevantes para los miembros.

- Acceso a sitios de Internet operados por moderadores de red o por personas suscritas que brindan información relevante para sus miembros

- Acceso a grandes cantidades de información, en ocasiones preseleccionadas alrededor de un tema de interés común.

Para conformar esta red, PIT desarrolló una plataforma bilingüe virtual ${ }^{3}$ utilizando el Internet y facilitando a los actores de las distintas redes que conforman TAP intercambiar sus fortalezas y debilidades.

El resultado es que TAP, gracias a este proyecto ha logrado desarrollar los vínculos entre los actores de las distintas redes de telecentros, fundamentalmente operadores, facilitando de este modo el proceso de creación de una Red de Conocimiento global del movimiento de telecentros panamericano y, lo que es más importante, generando los medios para que estos actores sean capaces de elaborar proyectos conjuntos que les permitan mejorar las condiciones de sus respectivas comunidades y alcanzar objetivos comunes.

El proyecto se dividió en cuatro etapas de implementación:

1. Desarrollo de la plataforma y comunicación al interior de las distintas redes.

2. Registro de participantes en el proyecto y propuestas de intercambio.

3. Selección las mejores propuestas.

4. Viajes e intercambios virtuales entre los participantes seleccionados.

La primera etapa incluyó el desarrollo del espacio de encuentro de los actores de las distintas redes. (Un sitio Web dinámico diseñado con lenguaje PHP con una base de datos diseñada en MySQL y servidor IIS).

\section{Gráfico1: Pantalla de la plataforma del PIT.}

Durante esta etapa cabe destacar la coordinación entre las distintas redes, se creó un comité directivo con 
representantes de todas las organizaciones que forman TAP lo que permitió trabajar de forma conjunta en los materiales de comunicación para dar a conocer el PIT a los participantes. Se utilizó el idioma ingles para guiar los trabajos de coordinación.

La comunicación con los operadores y miembros del movimiento de telecentros no resultó sencilla, la novedad de la iniciativa dificultó la comprensión del concepto por parte de algunos actores y se preciso una labor de acompañamiento y guía para facilitar la participación.

La segunda etapa fue la que permitió poner en contacto a las personas de las distintas redes que conforman TAP. Durante 11 semanas todos los miembros de las redes tuvieron la oportunidad de informarse, registrarse en el programa y realizar sus propuestas de intercambio conjuntas con cualquier otro miembro. En este periodo se evidenció que gran parte de las bases del movimiento de telecentros no cuenta con las habilidades necesarias para realizar proyectos conjuntos basados en red y que PIT ha supuesto un gran cambio para todos los participantes que a lo largo del proceso fueron adquiriendo dichas habilidades para alcanzar un objetivo concreto trabajando con personas distantes geográficamente con las que formularon una propuesta con el apoyo de los tutores del PIT.

Se optó por un registro a modo de mercado, en el que cada persona tuvo la oportunidad de realizar una oferta de conocimiento o presentar una solicitud a modo de necesidad, esto permitió al resto de participantes identificar a sus potenciales socios para elaborar una propuesta de intercambio. Este formato fue útil para que los participantes identificasen a sus socios pero en realidad se comprobó que no existían participantes que ofrecían o recibían experiencia o conocimiento sino que en todas las propuestas que se ejecutaron los participantes recibieron y dieron en igual medida, por lo que el PIT se convirtió en una experiencia en la que los participantes actuaron de forma horizontal en el intercambio de su experticia.

Los y las participantes tuvieron que redactar su propuesta a modo de proyecto siguiendo un modelo sencillo pero que contenía todos los elementos requeridos por cualquier donante a la hora de formular un proyecto, es decir: antecedentes, objetivos a corto y largo plazo, actividades, presupuesto, beneficio/s y un breve resumen del proyecto.

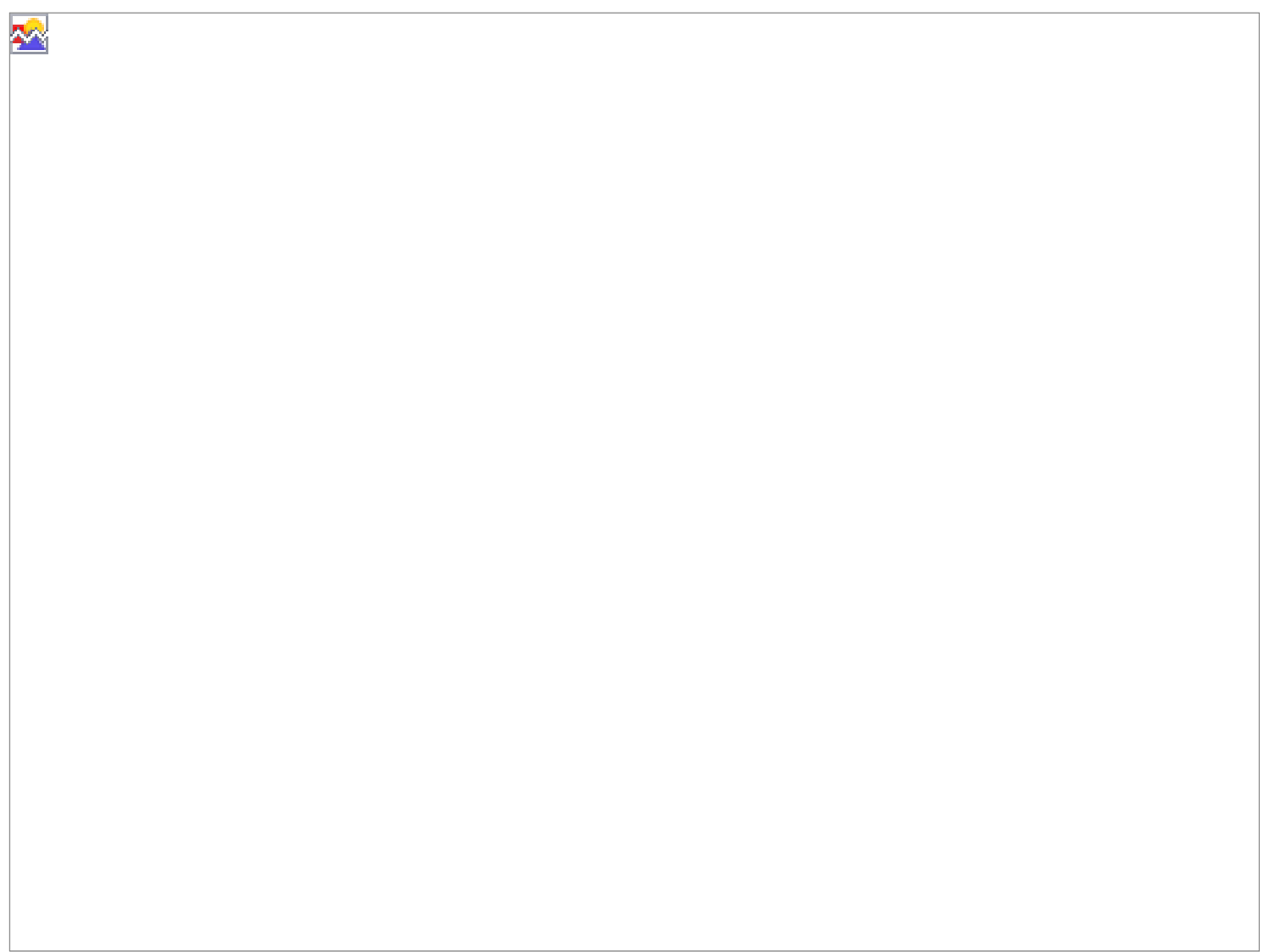

\section{Gráfico 2: Pantalla del mercado del PIT}

La participación en el PIT teniendo en cuenta el carácter piloto fue bastante elevada. Los coordinadores identificaron algunos factores que pueden facilitar y mejorar la participación de cara a futuras ediciones que se detallan a continuación:

- Novedad. PIT ha sido un proyecto piloto. En TAP no existían precedentes de actividades que beneficien directamente a los operadores de los telecentros.

- Falta de capacitación en la elaboración de propuestas. Se detecto una baja preparación para la formulación 
de proyectos.

- Falta de acceso eficaz a Internet. Este es un recurso que todavía no es estable en muchos telecentros, especialmente en América Latina y el Caribe.

- Falta de una cultura del uso del Internet como herramienta para la elaboración de propuestas conjuntas.

- Timidez y miedo. Algunos operadores expresaron temor a participar en este tipo de actividades en la que debían compartir con la comunidad de telecentros sus necesidades o sus ofertas. En otros casos se observó cierta timidez que reprimió su participación.

- Falta de apoyo institucional a los operadores. Algunos telecentros son apoyados por ONGs u otros organismos que mantienen el telecentro como un proyecto de su exclusividad no permitiendo a los operadores participar en este tipo de actividades.

Asimismo se identificaron unos roles básicos que debe cumplir la coordinación de este tipo de proyectos y que detallamos a continuación:

- Motivar a los individuos a participar activamente.

- Brindar asistencia permanente

- Comunicación clara, sistemática y permanente a través de distintos medios (virtuales y físicos).

Al final del proceso de inscripción se realizaron 13 propuestas de intercambio entre los participantes, todas ellas de gran calidad. Les fue asignado un tutor por el PIT que les ayudó a resolver sus dudas y orientar mejor sus propuestas.

Un jurado seleccionó las ocho mejores propuestas (ver tabla 2) que contaron con el apoyo financiero para que pudieran implementar sus intercambios dando paso así a la cuarta y ultima etapa de implementación del proyecto en la que los operadores tuvieron la oportunidad de viajar a otros países, dentro de sus países o realizar intercambios virtuales.

En el aspecto financiero cabe destacar que los montos para realizar este tipo de intercambios no son excesivamente costosos, tan sólo un proyecto supero los 5.000 dólares. Asimismo conviene señalar que los participantes realizaron sus aportaciones a los proyectos.

\begin{tabular}{|c|c|}
\hline Nombre de las propuestas ganadoras & Participantes \\
\hline $\begin{array}{l}\text { Colaboración e Intercambio de Conocimiento } \\
\text { Knowledge Exchange and Collaboration }\end{array}$ & $\begin{array}{l}\text { CAP Society of Cape Breton } \\
\text { County, Marieval } \\
\text { Enterprise Center Inc }\end{array}$ \\
\hline $\begin{array}{l}\text { Mejora de la comunicación entre los inmigrantes mayas y sus } \\
\text { familias a través de los telecentros. } \\
\text { Improving communication between Mayan immigrants and their } \\
\text { families with Telecenters }\end{array}$ & $\begin{array}{l}\text { CETEBI Comunitario Ajb'atz' } \\
\text { Enlace Quiche } \\
\text { CEDC/GNBC3 }\end{array}$ \\
\hline $\begin{array}{l}\text { Potenciar la Oferta Digital para 1@s Jóvenes de los programas } \\
\text { BiblioRedes de Chile y Poder Joven de México. } \\
\text { Enhancing Digital Offer for Youth from BiblioRedes (Chile) and } \\
\text { Poder Joven (Mexico) }\end{array}$ & $\begin{array}{l}\text { Red Nacional de Centros } \\
\text { Interactivos Poder Joven }\end{array}$ \\
\hline $\begin{array}{l}\text { Intercambio de experiencias para fortalecer el trabajo de las } \\
\text { comunidades indígenas en Sololá, Guatemala y en el Chaco, } \\
\text { Ecuador - principalmente fortalecer la capacidad de administración } \\
\text { de ambos Telecentros Comunitarios. } \\
\text { Exchange of experiences to strengthen the work of indigenous } \\
\text { communities in Sololá (Guatemala) and el Chaco (Ecuador) mainly }\end{array}$ & $\begin{array}{l}\text { Telecentro Comunitario el Chaco } \\
\text { Telecentro Asodigua }\end{array}$ \\
\hline
\end{tabular}




\begin{tabular}{|c|c|}
\hline \multicolumn{2}{|l|}{ the administrative capacity of both community telecenters. } \\
\hline $\begin{array}{l}\text { Intercambio para el fortalecimiento de la Escuela, Familia y } \\
\text { Comunidad, basado en el uso estratégico de las TIC en aplicaciones } \\
\text { educativas, con los telecentros comunitarios, para el desarrollo } \\
\text { local. }\end{array}$ & \\
\hline $\begin{array}{l}\text { Exchange for the strengthening of School, Family and Community } \\
\text { based in the strategic use of ICTs in education through community } \\
\text { telecenters. }\end{array}$ & \\
\hline Intercambiar experiencias entre jóvenes & $\begin{array}{l}\text { TELECENTRO WUAITA NAPO } \\
\text { - ECUADOR }\end{array}$ \\
\hline Exchange of experiences between young people. & Escuela Politécnica Nacional \\
\hline Prevención y atención de desastres naturales desde telecentros. & Telecentro Asodigua \\
\hline $\begin{array}{l}\text { Prevention and attention of natural disasters through community } \\
\text { telecenters. }\end{array}$ & CORTEN \\
\hline $\begin{array}{l}\text { Acceso inalámbrico para crear oportunidades para el desarrollo } \\
\text { económico comunitario. }\end{array}$ & SWSDA \\
\hline $\begin{array}{l}\text { Wireless WAN creates opportunities for Community Economic } \\
\text { Development }\end{array}$ & Marieval Enterprise Center Inc \\
\hline TOTAL & \\
\hline
\end{tabular}

Tabla 2: Propuestas ganadoras y presupuesto

Gráfico 3: Países receptores de experticia de las propuestas ganadoras 


\section{Gráfico 4: Países oferentes de experticia de las propuestas ganadoras}

En los gráficos 3 y 4 se detallan los países a los que pertenecen los participantes de las propuestas ganadoras. El grafico 3 detalla los países que se registraron como receptores de experticia y en el 4 los que se registraron como oferentes de la misma, aunque como ya se señalo con anterioridad al final del proceso se observó que no se cumplió con tal papel sino que todos los participantes intercambiaron experticia de modo horizontal. Destaca la participación de Ecuador y Canadá que fueron los países a los que perteneció la coordinación sur y norte del programa. Esto sin duda facilito la promoción personal de PIT y dio lugar a un mayor número de propuestas de ambos países.

De las ocho propuestas ganadoras tan sólo una planteó alcanzar sus objetivos a través de un medio completamente virtual, el resto requirieron viajar para el intercambio de conocimiento y tan solo una propuesta se realizó entre dos países con idiomas distintos lo que nos apunta a que el idioma puede ser una barrera para determinados intercambios.

Todas las propuestas han sido de gran valor, en este artículo no se analizan individualmente las mismas ya que se requeriría de una extensión mucho mayor para presentar las mismas, sin embargo son muchas las lecciones y resultados de este programa, a continuación se detalla una síntesis de las mismas.

\section{Resultados del proyecto.}

A continuación se presentan un resumen de los resultados alcanzados a tres niveles: por parte de los participantes en el PIT, por parte de las comunidades beneficiadas por los telecentros y finalmente por parte de las redes que conforman TAP.

Participantes:

- Capacidad de trabajo en red sin fronteras con otros operadores de telecentros.

- Capacidad de elaborar propuestas de proyecto.

- Identificación de actividades y modelos de telecentros innovadores en los países anfitriones que fueron aplicados en sus telecentros de origen.

- Fortalecimiento del compromiso personal con el movimiento de telecentros.

- Creación de relaciones personales transnacionales que han dado como resultado un incremento del intercambio informal de información en TAP.

Comunidades:

- Implementación de modelos innovadores adaptados de las visitas a otros telecentros.

- Creación de relaciones organizativas transnacionales.

Redes de telecentros (TAP):

- Fortalecimiento del trabajo en red, el intercambio de conocimiento y la cooperación entre las redes.

- Creación de contenido sobre programas y propuestas innovadoras disponibles a través del sitio Web de TAP y listas de discusión y otros medios de las redes participantes.

- Creación de un modelo de programa de intercambio entre telecentros que puede ser alojado e implementado por una red de telecentros fácilmente replicable y adaptable a otras realidades. 
Finalmente en una mirada hacia los resultados alcanzados individualmente por las distintas propuestas cabe destacar la apertura de un nuevo telecentro en la localidad de Challhuahuacho (Perú) fruto de los intercambios realizados gracias al PIT; este telecentro además será un espacio muy ligado a la educación gracias a los conocimientos adquiridos durante el intercambio en la pedagogía constructivista que la Fundación ChasquiNet esta aplicando en su programa "Escuelas Inter@ctivas" en Ecuador. Los acuerdos alcanzados entre las alcaldías de El Chaco (Ecuador) y San José de Chacaya (Guatemala) permitirán a las comunidades mejorar sus ingresos económicos utilizando sus telecentros como vitrinas para promocionar y comercializar sus productos.

Estos son solo algunos ejemplos puntuales de lo que este proyecto piloto ha alcanzado, en siguientes fases esperamos que el PIT pueda seguir fortaleciendo el movimiento de telecentros y alcanzar muchos más resultados.

\section{Conclusión}

El PIT ha sido un primer e importante paso que ha demostrado una posible evolución de las redes de telecentros de simples espacios de apoyo hacia redes de trabajo colaborativo, un concepto que en el movimiento de telecentros puede beneficiar a un gran numero de actores a los que representan los telecentros en sus respectivas comunidades, en este sentido las comunidades se extienden de manera global en lo que denominamos comunidades transnacionales.

A lo largo del articulo hemos visto como esta experiencia piloto ha demostrado la viabilidad de este tipo de experiencias en el movimiento de telecentros y su pertinencia para los operadores, un grupo de gran importancia en el movimiento de telecentros que no siempre cuenta con el apoyo necesario para desempeñar su trabajo y mejorar sus capacidades.

En un mundo cada vez más globalizado los telecentros enfrentan el desafío de ayudar a transformar no solo su comunidad sino la percepción que tenemos de la propia globalización, un fenómeno cambiante que requiere construir una visión común por parte del movimiento de telecentros desde las múltiples diferencias que conforman el continente americano y sus comunidades.

Esa globalización no es ajena a este movimiento, TAP es una red de redes que agrupa a los distintos actores del movimiento de telecentros panamericano, pero la globalización no se detiene en este continente y ya esta en marcha el proyecto de una red global a nivel mundial: Global Telecenter Alliance (GTA) una red totalmente global que une a los distintos movimientos y redes de telecentros de todo el planeta del que TAP forma parte y que permitirá que este tipo de proyectos eliminen las fronteras físicas tal y como las conocemos hoy en día, facilitando el desarrollo humano y social a través de los telecentros. 


\section{Bibliografía}

CANALES A. ZLONISKI, Ch. (2000). Comunidades y Migración en la era de la Globalización. Ponencia presentada en el Simposio sobre Migración Internacional en las Américas. San José, Costa Rica, 4 al 6 de Septiembre de 2000.

DELGADILLO, Karin, GÓMEZ, Ricardo y STOLL, Klaus (2002). Telecentros... ¿Para qué? Lecciones sobre telecentros comunitarios en América Latina y el Caribe. (Primera edición). Centro Internacional de Investigaciones para el Desarrollo, CIID: Canadá.

GUIKT, Irene y WOODHILL, Jim (2005) El aprendizaje a través de Redes Electrónicas y Temas Relacionados a Monitoreo y Evaluación. Descargado el 12 de febrero de 2007 de: www.grupochorlavi.org/php/doc/documentos/aprendizajemonitoreo.pdf

PROENZA, BASTIDAS Y MONTERO. (2004) Telecentros para el desarrollo socioeconómico y rural en América Latina. Documento de trabajo. FAO. Centro de inversiones. UIT. Sector de desarrollo de las telecomunicaciones. BID. División de Programas Sociales, Departamento de Operaciones 2, Unidad Rural, Departamento de Desarrollo Sostenible, Unidad de Tecnología de Información para el Desarrollo: Washington, D.C. Estados Unidos.

ROESSNER, Christoph. (2005). ICT and Development. The IT-infrastructure telecentre. Descargado en febrero de 2006 de: www.informatik.uni-hamburg.de/publications/papers/Dipl/Roessner_The_ITinfrastructure_telecentre.pdf

TAP (2006), Inter-American Telecenter Practitioner Exchange Program (IT-PEP). Documento de proyecto aprobado por IDRC (Canadá) en mayo de 2006. 